\title{
Effects of Electrical Stimulation Rate on Speech Recognition in Cochlear Implant Users
}

\author{
Sung Hye Park, Eunoak Kim, Hyo-Jeong Lee and Hyung-Jong Kim \\ Department of Otolaryngology, Hallym University College of Medicine, Chuncheon, Korea
}

Received August 25, 2011

Revised November 20, 2011

Accepted November 25, 2011
Background and Objectives: The stimulus signals delivered in cochlear implant (Cl) systems are generally derived by sampling the temporal envelope of each channel at some constant rate and using its intensity to control the stimulation current level delivered to the corresponding electrode site. The objective of the study was to investigate speech recognition performance of cochlear implant users in quiet and noisy environments using either moderate or high rates of electrical stimulations. Materials and Methods: Six post-lingually deafened adult users of the Nucleus $\mathrm{Cl} 24$ cochlear implant (Contour ${ }^{\circledR}$ electrode array, Cochlear ${ }^{\mathrm{TM}}$, Macquarie Park, Australia) with the Freedom ${ }^{\circledR}$ speech processor participated in the study. Stimulation rates of 900 and 2400 pulses-per-second/channel (pps/ch) were used after both stimulation programs were balanced for loudness. Monosyllabic word and sentence recognition scores in quiet and noisy environments were evaluated for each stimulation program after two months of practice. Subjects were also asked to respond to a questionnaire to examine their preference to any stimulation rate in different hearing conditions. Results: Word recognition scores for monosyllabic words in quiet conditions with the 900 stimulation rate was better than that of the 2400 stimulation rate, although no significant differences between them were found for sentence test in noise. A survey questionnaire indicated that most subjects preferred the 900 stimulation rate to the 2400 stimulation rate, especially in quiet conditions. Conclusions: Most subjects indicated a preference for $900 \mathrm{pps} / \mathrm{ch}$ rate in quiet conditions. It is recommended to remap at $900 \mathrm{pps} / \mathrm{ch}$ for those $\mathrm{Cl}$ users whose performance in quiet conditions is less than ideal.

Korean J Audiol 2012;16:6-9

\section{Introduction}

Cochlear implant (CI) users perceive sounds by the temporal envelope that is determined by the amplitude and rate of the electrical current created from each microelectrode channel. The electrical stimulation rate can be adjusted during the process of mapping a speech processor after CI surgery. In theory, high rates of electrical stimulation allow more information in the envelope, so that it can enable CI users to achieve better speech recognition. ${ }^{1)}$

Clinically useful stimulation rates are divided by below 500 pulse per second/channel (pps/ch)(low), 500-1000 pps/ch (medium), and above $1000 \mathrm{pps} / \mathrm{ch}$ (high). The default parameter of the Sprint ${ }^{\circledR} 3 \mathrm{G}$ speech processor from Cochlear ${ }^{\mathrm{TM}}$ was set at $900 \mathrm{pps} / \mathrm{ch}$ in the past. Meanwhile, the company recommended $2400 \mathrm{pps} / \mathrm{ch}$ as their default parameter with launch- ing of the Freedom ${ }^{\circledR}$ speech processor, because studies reported that it is more effective to use higher stimulation rates in transferring speech information. However, other studies have reported that there was no difference in the speech recognition ability in terms of the stimulation rate. A number of studies have proposed that speech recognition is better with a $1200 \mathrm{pps} / \mathrm{ch}$ rate or even lower compared to higher rates such as $1800 \mathrm{pps} /$ ch. Afterwards, the default parameter of Freedom ${ }^{\circledR}$ was readjusted to $900 \mathrm{pps} / \mathrm{ch}^{2)}$ Other studies also reported that patients experienced more comfortable hearing with low to medium stimulation rates compared to ones higher than $1800 \mathrm{pps} / \mathrm{ch}^{3{ }^{3}}$

Very few studies have reported the relationship between the electrical stimulation rate and speech recognition ability in Korea. There are also controversies regarding which rate would be better in terms of speech recognition. ${ }^{4,5)}$ In this study, we compared speech recognition performance and subjective prefer- 
ences of CI adult users when using medium (900 pps/ch) or high (2400 pps/ch) electrical stimulation rates. First, word recognition performance was measured in quiet and noisy environments using different electrical stimulation rates. Second, a questionnaire was administered to assess preferences of electrical stimulation rates according to different listening environments.

\section{Materials and Methods}

\section{Subjects}

Six post-lingually deafened adult patients who were implanted with Nucleus CI24 (Contour ${ }^{\circledR}$ electrode array, Cochle$\operatorname{ar}^{\mathrm{TM}}$, Macquarie Park, Australia) with the Freedom ${ }^{\circledR}$ speech processor at Hallym University Sacred Heart Hospital participated in the study. All six patients had at least one year of CI experience (Table 1). This study was approved by the Institutional Review Board of our institute and followed the recommended guidelines.

\section{Method}

At the enrollment of CI users, electrical stimulation rates of $900 \mathrm{pps} / \mathrm{ch}$ and $2400 \mathrm{pps} / \mathrm{ch}$ were programmed as P1 and P2. For all participants, advanced combination encoders speech coding strategy with 22 channels was used as active electrodes for both programs. Stimulation mode was monopolar (MP)+1, with a maxima of 6-10 and a pulse width of $12-25 \mu$ s. Sound loudness level was controlled by mapping the device at a comfortable level using T and C levels for each participant. Participants were informed about the goal of the study and its procedures at their first visit. They were also instructed to use each stimulation program for two weeks alternatively two times each for a 2-month study period. After that, they were asked to answer a questionnaire to assess their preference to any stimulation rate, and monosyllabic word and sentence recognition scores in quiet and noisy conditions were evaluated for each stimulation program. To evaluate word recognition under a quiet condition, one out of four monosyllabic word lists (Korean Standard-Monosyllabic Word Lists for Adults), of which each was composed of 50 consonant-nucleus-consonant words were randomly selected. Subjects were asked to listen to stimuli at $45 \mathrm{~dB}$ hearing level (HL) from a compact disc at 0 degree speaker after calibration. The word recognition score calculated by the ratio of correctly perceived words in percentage was measured using two different stimulation rates. The sentence recognition test in noisy conditions was performed by presenting 10 sentences with white noise. A sentence list, including 10 sentences, was selected from eight sentence lists (Korean standard-sentence lists for adults), ${ }^{7)}$ of which each sentence included three to five keywords. Starting from $50 \mathrm{~dB}$ HL with a signal to noise ratio (SNR) of $15 \mathrm{~dB}$, the SNR was reduced when a correct response of key words was more than $70 \%$ and increased when less than $70 \%$ was recognized. The tests were performed alternatively for two stimulation rate conditions. ${ }^{6)}$ Test stimuli retrieved from the standardized Korean speech materials were presented through a compact disc. ${ }^{7)}$

Subjects were also asked to respond to a questionnaire and rate their preference on a scale of 1 to 5 in terms of stimulation rate under three listening conditions such as quiet and noisy environments and media (TV/radio) listening environment.

\section{Statistical analysis}

Statistical analysis was performed using SPSS 17.0 (SPSS Inc., Chicago, IL, USA). A Wilcoxon rank sum test was used at a significance level of 0.05 .

\section{Results}

\section{Word recognition score in quiet conditions and} sentence recognition in noisy conditions

When subjects underwent word recognition test in a quiet environment using $900 \mathrm{pps} / \mathrm{ch}$ and $2400 \mathrm{pps} / \mathrm{ch}$ stimulation rates, performance was significantly better with the 900 pps/ ch stimulation rate than with the $2400 \mathrm{pps} / \mathrm{ch}$ stimulation rate $(p=0.027)$ (Fig. 1).

For sentence test in a noisy environment, there was no significant difference in SNRs between stimulation rates ( $p=$ 0.684). However, three out of six subjects indicated higher

Table 1. Subject demographics

\begin{tabular}{ccccccc}
\hline Code & Sex/Age & Duration of device use (yrs) & Stimulation rate (pps/ch) & Maxima & Pulse width ( $\mu$ s) & Speech strategy \\
\hline A & F/17 & 6 & 900 & 8 & 25 & ACE \\
B & M/43 & 3 & 2400 & 10 & 12 & ACE \\
C & F/25 & 3 & 2400 & 6 & 8 & ACE \\
D & F/25 & 1 & 900 & 8 & 25 & ACE \\
E & M/13 & 1 & 900 & 8 & 25 & ACE \\
F & F/32 & 1 & 900 & ACE \\
\hline
\end{tabular}

ACE: advanced combination encoders 


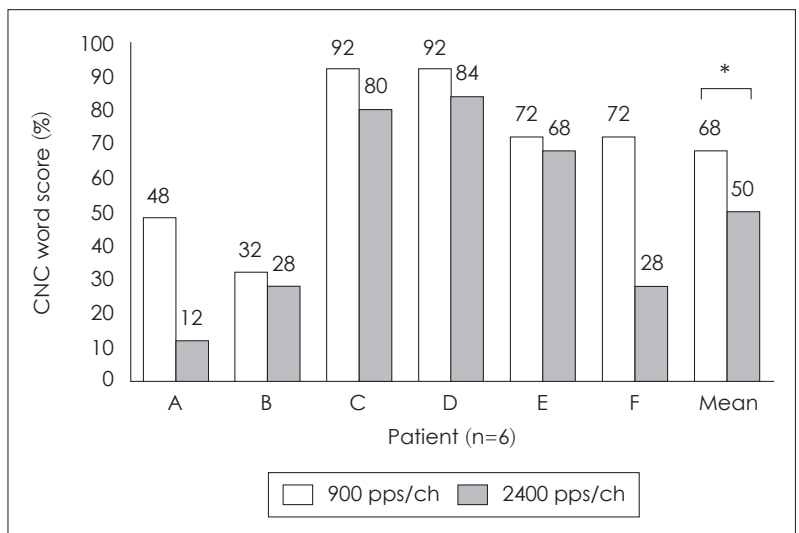

Fig. 1. A comparison of word recognition test results between two stimulation rate conditions using $\mathrm{CNC}$ monosyllables in quiet conditions. $* p<0.05$. CNC: consonant-nucleus-consonant.

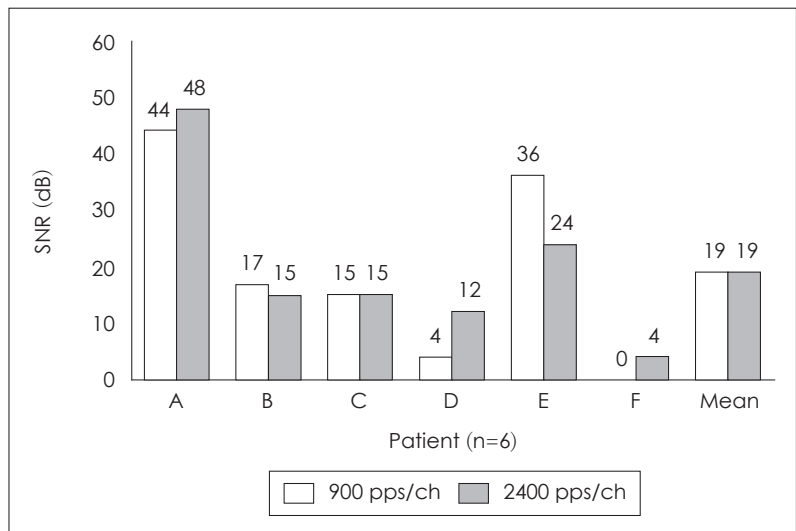

Fig. 2. A comparison of SNR from the sentence recognition test between two stimulation rate conditions in noisy conditions. SNR: signal to noise ratio.

SNRs when using the $2400 \mathrm{pps} / \mathrm{ch}$ stimulation rate, while two subjects showed higher SNRs when using the $900 \mathrm{pps} / \mathrm{ch}$ stimulation rate. Only one subject indicated the same SNRs in both stimulation rates (Fig. 2).

\section{Questionnaire}

When subjects were asked to respond to a questionnaire to examine their preference to any stimulation rate in quiet and noisy environments and media (TV/radio) listening conditions, they indicated that the $900 \mathrm{pps} / \mathrm{ch}$ stimulation rate was better in most listening settings. In particular, a significant difference was found in quiet and media (TV/radio) listening environments $(p<0.05)$ (Table 2).

When we assessed the degree of helpfulness for two types of stimulation rates in three listening environments, subjects showed that the $900 \mathrm{pps} / \mathrm{ch}$ stimulation rate was helpful in quiet and media (TV/radio) listening environments ( $p=0.023$ and 0.026). However, no significant difference was found in a noisy environment $(p>0.05)$ (Fig. 3).
Table 2. Subject preference in different listening conditions (quiet, noise, or media)

\begin{tabular}{ccc}
\hline Condition & $900 \mathrm{pps} / \mathrm{ch}(\mathrm{no} / \%)$ & $2400 \mathrm{pps} / \mathrm{ch}(\mathrm{no} / \%)$ \\
\hline Quiet & $6 / 100$ & $0 / 0$ \\
Noise & $4 / 66.6$ & $2 / 33.4$ \\
Media & $6 / 100$ & $0 / 0$ \\
\hline
\end{tabular}

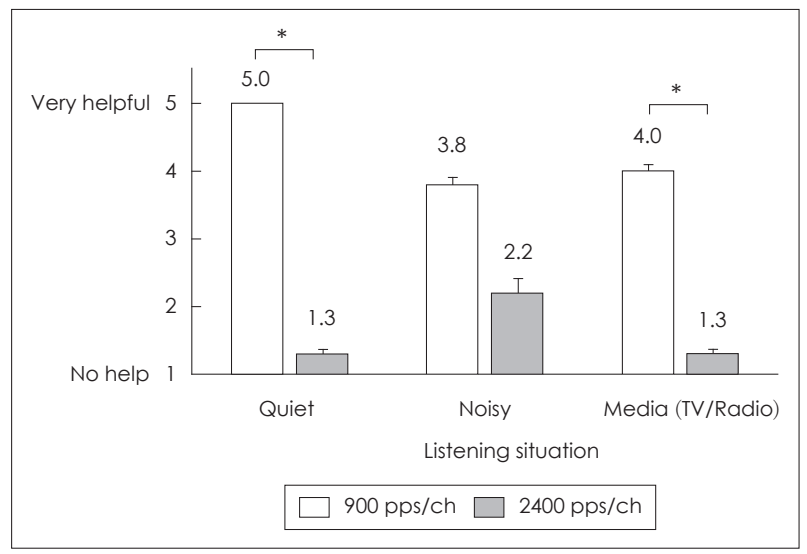

Fig. 3. Subjective helpfulness rating for different stimulation rates in quiet, noise, and media (TV/radio) listening situations. Rating 1 represents "no help", and rating 5 represents "very helpful". * $p<0.05$.

\section{Discussion}

Higher word recognition scores with the $900 \mathrm{pps} / \mathrm{ch}$ stimulation rate were achieved in a quiet environment compared to that of $2400 \mathrm{pps} / \mathrm{ch}$. Accordingly, the $900 \mathrm{pps} / \mathrm{ch}$ stimulation rate was preferred over $2400 \mathrm{pps} / \mathrm{ch}$ in terms of subjective preferences and the degree of helpfulness. Higher word recognition scores using an intermediate stimulation rate compared to high stimulation rates have been reported by previous studies that used other CI devices from Med-El and Advanced Bionics. ${ }^{8,9)}$ These studies reported that the $1615 \mathrm{pps} / \mathrm{ch}$ stimulation rate was preferred over $250 \mathrm{pps} / \mathrm{ch}$ and $810 \mathrm{pps} / \mathrm{ch}$ when listening to music or radio. However, our study showed preference for the $900 \mathrm{pps} / \mathrm{ch}$ stimulation rate. This may be due to the design of our study, in which we only used two stimulation rates of $900 \mathrm{pps} / \mathrm{ch}$ and $2400 \mathrm{pps} / \mathrm{ch}$. It is assumed that CI users in general prefer intermediate stimulation rates, such as $900 \mathrm{pps} / \mathrm{ch}$ or $1615 \mathrm{pps} / \mathrm{ch}$, instead of high stimulations such as $2400 \mathrm{pps} / \mathrm{ch}$ when listening to radio or music. ${ }^{4}$

Speech recognition in noise is generally evaluated at fixed SNRs, and scores were compared in different noise conditions. A recent study by Arora, et al. ${ }^{6}$ reported SNRs at which subjects achieved $70 \%$ of speech recognition. He compared stimulation rates of $275 \mathrm{pps} / \mathrm{ch}, 350 \mathrm{pps} / \mathrm{ch}, 500 \mathrm{pps} / \mathrm{ch}$, and 900 $\mathrm{pps} / \mathrm{ch}$, and the $900 \mathrm{pps} / \mathrm{ch}$ stimulation rate was the most preferred by most CI users. The purpose of mapping is to program the processor to deliver sounds and maximize the indi- 
vidual's ability to communicate in their everyday living. Even though the default parameters provide a comfortable listening environment, changes in the electrical stimulation rate may affect speech recognition in some specific hearing environments. It is recommended to adjust electrical stimulation parameters based on an individual's needs to provide an optimal listening environment for CI users. It is also important to evaluate performance under various environments, such as word recognition in quiet conditions and sentence test in noisy conditions, including a questionnaire of the device program preference to find an optimal setting for each user. ${ }^{10)}$ Two out of six subjects had been using the $2400 \mathrm{pps} / \mathrm{ch}$ stimulation rate before they were involved in our study. As indicated by the results of our current study, all CI users preferred $900 \mathrm{pps} / \mathrm{ch}$ and started using a program with the $900 \mathrm{pps} / \mathrm{ch}$ stimulation rate in their daily lives. It can be predicted that some CI users who are currently using the $2400 \mathrm{pps} / \mathrm{ch}$ stimulation rate can receive benefit from using either the $500 \mathrm{pps} / \mathrm{ch}$ or $900 \mathrm{pps} / \mathrm{ch}$ stimulation rate. In addition, using lower stimulation rates, such as 900 pps/ ch or $500 \mathrm{pps} / \mathrm{ch}$, will result in extended battery life compared to $2400 \mathrm{pps} / \mathrm{ch}^{11)}$ A previous study reported that CI users with tinnitus preferred higher stimulation rates. ${ }^{4)}$ Further studies will be performed to investigate the relationship between tinnitus and stimulation rates of the CI processor.

\section{Conclusion}

We investigated whether different stimulation rates affected performance in word recognition and sentence recognition in noisy conditions. Despite some limitations, such as the small number of subjects and stimulation rates, this study showed that adjustments of the stimulation rate may result in better speech listening, regardless of duration of use of a particular program. Further studies are necessary to investigate improve- ment with various stimulation rates using a larger subject group with prolonged use of CI.

\section{Acknowledgments}

This study was funded by a basic research project of the National Research Foundation of Korea (Grant number: 2011-0005657).

\section{REFERENCES}

1) Holden LK, Skinner MW, Holden TA, Demorest ME. Effects of stimulation rate with the Nucleus 24 ACE speech coding strategy. Ear Hear 2002;23:463-76.

2) Cochlear Ltd. Selecting stimulation rate with the Nucleus freedom system. White paper prepared by Cochlear Ltd.;2007.

3) Balkany T, Hodges A, Menapace C, Hazard L, Driscoll C, Gantz B, et al. Nucleus Freedom North American clinical trial. Otolaryngol Head Neck Surg 2007;136:757-62.

4) Vandali AE, Whitford LA, Plant KL, Clark GM. Speech perception as a function of electrical stimulation rate: using the Nucleus 24 cochlear implant system. Ear Hear 2000;21:608-24.

5) Dreschler WA, Verschuure H, Ludvigsen C, Westermann S. ICRA noises: artificial noise signals with speech-like spectral and temporal properties for hearing instrument assessment. International Collegium for Rehabilitative Audiology. Audiology 2001;40:148-57.

6) Arora K, Dawson P, Dowell R, Vandali A. Electrical stimulation rate effects on speech perception in cochlear implants. Int J Audiol 2009; 48:561-7.

7) Lee JH, Jo SJ, Kim JS, Jang HS, Lim DW, Lee KW. Korean Speech Audiometry. Hakjisa;2010.

8) Verschuur CA. Effect of stimulation rate on speech perception in adult users of the Med-El CIS speech processing strategy. Int J Audiol 2005;44:58-63.

9) Plant KL, Whitford LA, Psarros CE, Vandali AE. Parameter selection and programming recommendations for the ACE and CIS speech-processing strategies in the Nucleus 24 cochlear implant system. Cochlear Implants Int 2002;3:104-25.

10) Friesen LM, Shannon RV, Cruz RJ. Effects of stimulation rate on speech recognition with cochlear implants. Audiol Neurootol 2005; 10:169-84.

11) Weber BP, Lai WK, Dillier N, von Wallenberg EL, Killian MJ, Pesch $\mathrm{J}$, et al. Performance and preference for ACE stimulation rates obtained with nucleus RP 8 and freedom system. Ear Hear 2007;28(2 Suppl):46S-8S. 